\title{
Plasma glial fibrillary acidic protein and neurofilament light chain for the diagnostic and prognostic evaluation of frontotemporal dementia
}

Nuole Zhu 1,2,3, Miguel Santos-Santos ${ }^{1,2}$, Ignacio Illán-Gala1,2, Victor Montal ${ }^{1}$, Teresa Estellés ${ }^{1,2}$, Isabel Barroeta ${ }^{1,2}$, Miren Altuna ${ }^{1,2}$, Javier Arranz ${ }^{1,2}$, Laia Muñoz ${ }^{2}$, Olivia Belbin ${ }^{1,2}$, Isabel Sala ${ }^{1,2}$, Maria Belén Sánchez-Saudinós $s^{1,2}$, Andrea Subirana ${ }^{1,2}$, Laura Videla ${ }^{1,2,4}$, Jordi Pegueroles ${ }^{1}$, Rafael Blesa' ${ }^{1}$, Jordi Clarimón ${ }^{1,2}$, Maria Carmona-Iragui ${ }^{1,2,4}$, Juan Fortea ${ }^{1,2,4}$, Alberto Lleó ${ }^{1,2,3^{*}}$ and Daniel Alcolea ${ }^{1,2,3^{*}}$ (1)

\begin{abstract}
Background: Astrocytes play an essential role in neuroinflammation and are involved in the pathogenesis of neurodenegerative diseases. Studies of glial fibrillary acidic protein (GFAP), an astrocytic damage marker, may help advance our understanding of different neurodegenerative diseases. In this study, we investigated the diagnostic performance of plasma GFAP (pGFAP), plasma neurofilament light chain ( $\mathrm{pNfL}$ ) and their combination for frontotemporal dementia (FTD) and Alzheimer's disease (AD) and their clinical utility in predicting disease progression.

Methods: pGFAP and pNfL concentrations were measured in 72 FTD, 56 AD and 83 cognitively normal (CN) participants using the Single Molecule Array technology. Of the 211 participants, 199 underwent cerebrospinal (CSF) analysis and 122 had magnetic resonance imaging. We compared cross-sectional biomarker levels between groups, studied their diagnostic performance and assessed correlation between CSF biomarkers, cognitive performance and cortical thickness. The prognostic performance was investigated, analyzing cognitive decline through group comparisons by tertile.
\end{abstract}

Results: Unlike pNfL, which was increased similarly in both clinical groups, pGFAP was increased in FTD but lower than in $\mathrm{AD}$ (all $P<0.01$ ). Combination of both plasma markers improved the diagnostic performance to discriminate FTD from AD (area under the curve [AUC]: combination 0.78; pGFAP 0.7; pNfL 0.61, all $P<0.05$ ). In FTD, pGFAP correlated with cognition, CSF and plasma NfL, and cortical thickness (all $P<0.05$ ). The higher tertile of pGFAP was associated with greater change in MMSE score and poor cognitive outcome during follow-up both in FTD (1.40 points annually, hazard ratio $[H R] 3.82, P<0.005)$ and in AD (1.20 points annually, HR 2.26, $P<0.005)$.

Conclusions: pGFAP and pNfL levels differ in FTD and AD, and their combination is useful for distinguishing between the two diseases. pGFAP could also be used to track disease severity and predict greater cognitive decline during follow-up in patients with FTD.

\footnotetext{
*Correspondence: alleo@santpau.cat; dalcolea@santpau.cat

${ }^{1}$ Sant Pau Memory Unit, Department of Neurology, Institut

d'Investigacions Biomèdiques Sant Pau - Hospital de Sant Pau, Universitat

Autònoma de Barcelona, 08041 Barcelona, Spain

Full list of author information is available at the end of the article
}

(c) The Author(s) 2021. Open Access This article is licensed under a Creative Commons Attribution 4.0 International License, which permits use, sharing, adaptation, distribution and reproduction in any medium or format, as long as you give appropriate credit to the original author(s) and the source, provide a link to the Creative Commons licence, and indicate if changes were made. The images or other third party material in this article are included in the article's Creative Commons licence, unless indicated otherwise in a credit line to the material. If material is not included in the article's Creative Commons licence and your intended use is not permitted by statutory regulation or exceeds the permitted use, you will need to obtain permission directly from the copyright holder. To view a copy of this licence, visit http://creativecommons.org/licenses/by/4.0/. The Creative Commons Public Domain Dedication waiver (http://creativeco mmons.org/publicdomain/zero/1.0/) applies to the data made available in this article, unless otherwise stated in a credit line to the data. 
Keywords: Glial fibrillary acidic protein, Neurofilament, Frontotemporal dementia, Plasma biomarkers

\section{Introduction}

Frontotemporal dementia (FTD) is a progressive neurodegenerative condition characterized by clinical, genetic and neuropathologic heterogeneity. The clinical manifestation of FTD may overlap with psychiatric or other neurodegenerative disorders, such as Alzheimer's disease (AD). Diagnosis is thus a clinical challenge.

In the past two decades, many efforts have been made to find imaging or fluid biomarkers for FTD [1-6]. In spite of the notable advances, we still do not have pathophysiological markers of FTD to be used in clinical practice. Recently, some studies have shown that the concentrations of neurofilament light chain (NfL), a marker of axonal damage, are increased in the cerebrospinal fluid (CSF) and blood of FTD patients [6]. Moreover, their levels can reflect disease severity and predict clinical progression [1-6]. However, NfL is not specific for FTD and can be increased in other neurodegenerative diseases, such as AD and Lewy body dementia [6]. There is a considerable overlap of NfL level between different conditions [1-6].

Neuroinflammation and neurodegeneration are highly interrelated processes in neurological diseases [7]. Increased levels of astrogliosis marker glial fibrillary acidic protein (GFAP) in CSF and plasma, have been described in different neurodegenerative diseases [8-19, 20, 23-27]. In AD, recent studies showed that the plasma GFAP (pGFAP) levels are associated with amyloid pathology [15-18]. Furthermore, some studies have suggested that GFAP could be a marker of disease severity [8-22] and be a prognostic marker for progression to AD dementia in cognitively normal older adults [15-22]. Likewise, other studies have reported increased CSF GFAP and pGFAP concentrations in FTD, even though the results are not consistent across studies $[10,12,13$, 23-27]. Although previous studies did not detect changes of blood GFAP level in FTD [10, 12, 13], some studies have observed elevated pGFAP in all different FTD subgroups $[24,25,26]$. Higher pGFAP concentrations have been associated with greater functional impairment and disease severity in two studies $[13,25]$, but the role of pGFAP in predicting disease progression of FTD and its potential use for diagnosis, either alone or in combination with other biomarkers, remain unclear.

In this study, our primary research aim was to determine the potential use of pGFAP, pNfL or their combination to distinguish patients with FTD from AD and from cognitively normal participants $(\mathrm{CN})$, and to study their association with disease progression in FTD. The secondary aim was to study the correlation of pGFAP with cognition, other biomarkers, and structural measures in neuroimaging.

\section{Methods}

\section{Study participants and classification}

We collected clinical and biomarker information of 211 participants with available plasma samples from the Sant Pau Initiative on Neurodegeneration (SPIN) cohort, a multimodal biomarker platform for the study of neurodegenerative diseases [28]. The participants were classified into the following clinical groups according to the internationally accepted diagnostic criteria: 72 patients with probable FTD-related clinical syndromes: 33 patients with behavioral variant of frontotemporal dementia (bvFTD) [29], 7 semantic variant of primary progressive aphasia (svPPA) [30], 14 nonfluent variant of primary progressive aphasia (nfvPPA) [30] and 18 progressive supranuclear palsy-corticobasal syndrome spectrum (PSP-CBD) [31, 32]. CSF AD biomarkers and/or amyloid PET were taken into account during the diagnostic classification of FTD patients, and those with positive AD biomarkers were excluded from this group. We also included $56 \mathrm{AD}$ patients with evidence of AD pathophysiology [33] either through CSF biomarkers $(n=51)$ or amyloidPET $(n=5)$, and $83 \mathrm{CN}$ that had normal CSF values of $\mathrm{A} \beta_{42} / \mathrm{A} \beta_{40}$ and pTau181, and neuropsychological evaluation within normal range [28].

Plasma availability was the prerequisite to include the participants in this study. CSF samples were available from 199 participants (94\%). A subset of 122 participants (58\%) underwent 3 Tesla structural brain MRI. APOE genotype was available in 203 participants (96\%), and 202 participants (96\%) were longitudinally followed up and underwent a comprehensive evaluation. All participants had a Mini-Mental State Examination (MMSE) score and a Global Deterioration Scale of Reisberg score at the time of diagnosis, and repeated measures of MMSE were obtained during the follow-up. In a subset of 45 FTD patients, Frontotemporal Dementia Rating Scale (FTDFRS) was available at the time of diagnosis.

\section{Blood and CSF sample analysis}

Blood samples were collected in 10-ml EDTA tubes and immediately transferred to our laboratory where they were centrifuged and aliquoted within $2 \mathrm{~h}$ after collection. CSF samples were collected on the same day of blood extraction and processed in polypropylene tubes following international recommendations [33]. All 
samples were processed and aliquoted within the first two hours after lumbar puncture. The plasma and CSF aliquots were stored at $-80{ }^{\circ} \mathrm{C}$ until analysis. pGFAP and pNfL concentrations were measured using the SR-X single molecule array. CSF AD core biomarkers (A $\beta 42$, $\mathrm{A} \beta 40$, tTau and pTau181) were measured in the fullyautomated platform Lumipulse (Fujirebio-Europe), and levels of CSF NfL (Uman Diagnostics) and CSF YKL-40 (MicroVue $^{\mathrm{TM}}$, Quidel) were measured by ELISA according to previously reported methods [7,33]. All samples and calibration curves were measured in duplicate. Intra- and inter-assay coefficients of variations were $5.3 \%$ and $9.6 \%$, respectively. The pre-analytical processing protocol for blood and CSF in the SPIN cohort has been described in detail $[28,29]$.

\section{Statistical analysis}

Chi-square test was used to compare sex and $A P O E \varepsilon 4$ genotype frequency between the groups. Continuous variables are expressed as means and standard deviation. Distributions of demographic and biomarker data were assessed using Shapiro-Wilk's test and homogeneity of variances was checked by Levene's test. Biomarker raw values not following a normal distribution were logtransformed to achieve a normal distribution. Age- and sex-adjusted analysis of covariance (ANCOVA) followed by post-hoc Tukey's test was used to compare the pGFAP and pNfL levels between groups. The potential use of pGFAP, pNfL or their combination to distinguish patients with FTD from $A D$ and $C N$ was assessed through areas under the curve (AUC) by receiver operating characteristic (ROC) analyses. ROC curves were compared using DeLong's test. To study the association of pGFAP with disease progression, we divided participants in 3 tertile groups according to their pGFAP levels. A linear-mixed model was used to assess the association of pGFAP with cognitive decline during the follow-up. We included age, sex, MMSE score at the time of diagnosis, pGFAP tertile and its interaction with time as fixed factors, and modeled random intercepts and slopes at the participant level to account for repeated measures. Multivariate Cox regression analysis adjusting for sex and baseline age and Kaplan Meier curves were performed to analyze the predictive value of pGFAP for significant cognitive impairment (MMSE score $<20$ ). To investigate the relationship of pGFAP level with demographics, cognitive scores, CSF biomarkers and pNfL level, Spearman's correlations were assessed. Cortical thickness was computed with Freesurfer software (version 5.1) [35] (https://surfer.nmr.mgh. harvard.edu/fswiki), and its correlation with pGFAP was assessed using the general linear model, including age and sex as fixed factors.
Statistical significance for all tests was set at 5\% $(P=0.05)$ and corrected for multiple comparisons. All statistical analyses were performed using packages "psych" (v. 2.0.8), "ggplot2" (v. 3.3.3), "pROC" (v. 1.16.2), "ImerTest" (v. 3.1-3), "nlme" (v. 3.1-148), "multcomp" (v. 1.4-13), "survival" (v. 3.2-11) and "survminer" (v. 0.4.9) as implemented in $\mathrm{R}$ statistical software version $\mathrm{R}$ 4.0.2 (http://www.R-project.org).

\section{Results \\ Demographics and clinical data}

Table 1 shows the demographics and clinical data, CSF and plasma biomarker levels in the FTD, $\mathrm{AD}$ and $\mathrm{CN}$ groups. Controls were significantly younger than symptomatic patients, but there was no difference in age between the symptomatic groups. Controls had more years of education than the symptomatic groups, and there were no differences in sex between the groups. $A P O E \varepsilon 4$ genotype was more frequent in the AD group. Both baseline and last MMSE scores were lower in the disease groups compared with the control group. Within the FTD subgroups, the baseline and last MMSE scores were lower in svPPA compared to other subgroups. The mean follow-up period was 3.4 $( \pm 2.3)$ years in FTD, 3.9 $( \pm 2)$ in $\mathrm{AD}$ and $4( \pm 1.7)$ in $\mathrm{CN}$. The follow-up period did not differ between the groups.

Age correlated with the pGFAP level $(\mathrm{Rho}=0.35$, $P<0.001)$ in the control group, but not with the pNfL level. No difference in pGFAP level was found between male and female participants. pNfL levels were higher in males in the whole sample $(P=0.05)$ and in the AD group $(P=0.04)$, but not in the $\mathrm{CN}$ or the FTD group.

\section{Plasma GFAP and NfL concentrations differ between FTD and $A D$}

After adjusting for age and sex, pGFAP and pNfL levels differed between FTD and AD patients (Fig. 1). The level of pGFAP was higher in patients with FTD $(234.9 \pm 141.9 \mathrm{pg} / \mathrm{ml})$ than in $\mathrm{CN}(134.3 \pm 45.4 \mathrm{pg} / \mathrm{ml}$, $P=0.0008)$ but lower than in AD $(319.8 \pm 135.1 \mathrm{pg} / \mathrm{ml}$, $P<0.001)$. Within the FTD subgroups, the pGFAP level was significantly higher in svPPA and PSP-CBD compared with controls $(P=0.03)$, and significantly lower in bvFTD than in $\mathrm{AD}(P<0.001)$ (Fig. 1a, b).

As expected, the pNfL levels were higher in both FTD $(37.6 \pm 42.3 \mathrm{pg} / \mathrm{ml}, P<0.001)$ and $\mathrm{AD}(26.5 \pm 13.3 \mathrm{pg} / \mathrm{ml}$, $P=0.004)$ groups compared to $\mathrm{CN}(18 \pm 20.2 \mathrm{pg} / \mathrm{ml})$. The pNfL levels were increased in all FTD subgroups $(P<0.05)$ compared to $\mathrm{CN}$, except in svPPA, which showed a similar trend as other FTD subgroups but did not reach statistical significance (Fig. 1c, d). 
Table 1 Demographics and clinical characteristics, CSF and plasma biomarker concentrations in CN, FTD and AD groups

\begin{tabular}{|c|c|c|c|c|c|c|c|}
\hline & \multirow[t]{2}{*}{$\mathrm{CN}$} & \multicolumn{5}{|l|}{ FTD } & \multirow[t]{2}{*}{$A D$} \\
\hline & & bvFTD & nfvPPA & svPPA & PSP-CBD & All FTD & \\
\hline$n$ & 83 & 33 & 14 & 7 & 18 & 72 & 56 \\
\hline Age, years & $58(8.5)^{\mathrm{b}, \mathrm{c}}$ & $68.3(10.6)^{\mathrm{a}}$ & $72.8(5.6)^{\mathrm{a}}$ & $73.1(11.1)^{\mathrm{a}}$ & $72.9(5.4)^{\mathrm{a}}$ & $70.8(8.9)^{\mathrm{a}}$ & $70.8(7.2)^{\mathrm{a}}$ \\
\hline \multicolumn{8}{|l|}{ Sex } \\
\hline Female & 49 & 10 & 9 & 4 & 11 & 34 & 31 \\
\hline Male & 34 & 23 & 5 & 3 & 7 & 38 & 25 \\
\hline \multicolumn{8}{|l|}{ APOE \&4 status } \\
\hline$\varepsilon 4+$ & $23^{c}$ & $3^{c}$ & $3^{c}$ & $2^{c}$ & $2^{c}$ & $10^{c}$ & $27^{a, b, d-g}$ \\
\hline$\varepsilon 4-$ & $57^{c}$ & $29^{c}$ & $11^{c}$ & $5^{c}$ & $14^{c}$ & $59^{c}$ & $27^{a, b, d-g}$ \\
\hline Follow up time, years & $3.9(1.7)$ & $4.1(2.5)$ & $3.1(2.3)$ & $4.8(1.7)$ & $1.9(1)$ & $3.4(2.3)$ & $4(2)$ \\
\hline Education, years & $15.4(4.1)^{b-g}$ & $12.9(5.6)^{\mathrm{a}}$ & $13.3(4.9)^{\mathrm{a}}$ & $14.8(5.2)^{\mathrm{a}}$ & $11.3(3.7)^{\mathrm{a}}$ & $12.7(5)^{\mathrm{a}}$ & $10.4(4.7)^{\mathrm{a}}$ \\
\hline Baseline MMSE score & $29.2(1)^{b-g}$ & $25.1(4.2)^{\mathrm{a}, \mathrm{f}}$ & $24.1(6.8)^{\mathrm{a}, \mathrm{f}}$ & $16.5(12.2)^{\mathrm{a}, \mathrm{c}-\mathrm{e}, \mathrm{g}}$ & $22.6(6.3)^{a, f}$ & $23.5(6.5)^{\mathrm{a}}$ & $23.7(3.8)^{\mathrm{a}}$ \\
\hline Last MMSE score & $29.3(1)^{b-g}$ & $16.6(10)^{a, f}$ & $14(12.2)^{\mathrm{a}, \mathrm{f}}$ & $0.4(0.9)^{a, c-e, g}$ & $11.6(12.4)^{\mathrm{a}}$ & $13.3(11.4)^{\mathrm{a}}$ & $15.5(9.5)^{\mathrm{a}}$ \\
\hline pGFAP, pg/ml & $134.3(45.4)^{b-d, f-g}$ & $193.1(100.1)^{\mathrm{a}}$ & $224.6(77.2)$ & $159.3(279.1)^{\mathrm{a}}$ & $271.2(148.2)^{\mathrm{a}}$ & $234.9(141.9)^{a, b}$ & $319.8(135.1)^{a, c}$ \\
\hline $\mathrm{pNfL}, \mathrm{pg} / \mathrm{ml}$ & $18(20.2)^{b-e, g}$ & $41.9(60.1)^{\mathrm{a}}$ & $34.9(17.2)^{\mathrm{a}}$ & $31.3(14.5)$ & $34.3(18.1)^{\mathrm{a}}$ & $37.6(42.3)^{\mathrm{a}}$ & $26.5(13.3)^{\mathrm{a}}$ \\
\hline CSF NfL, pg/ml & $494.8(274.3)^{b, c, f, g}$ & $1436.3(930.6)^{\mathrm{a}}$ & $1944.5(1146.7)$ & $2394.6(637.8)^{a}$ & $1739.3(1607.3)^{\mathrm{a}}$ & $1654.1(1187.6)^{\mathrm{a}, \mathrm{c}}$ & $1286.4(1136.9)^{a, b, f}$ \\
\hline CSF YKL-40, ng/ml & $190.3(49.4)^{\mathrm{b}, \mathrm{c}}$ & $265.8(64.7)^{\mathrm{a}}$ & $274.84(61)^{\mathrm{a}}$ & $311.8(37.1)^{\mathrm{a}}$ & $262.3(79.3)$ & $268.2(63.7)^{\mathrm{a}}$ & $287.3(68.1)^{\mathrm{a}}$ \\
\hline CSF A $\beta 42 / A \beta 40$ & $0.099(0.016)^{c}$ & $0.098(0.015)^{c}$ & $0.092(0.012)^{c}$ & $0.103(0.0002)^{c}$ & $0.095(0.012)^{c}$ & $0.097(0.013)^{c}$ & $0.046(0.0099)^{a, b, d-g}$ \\
\hline CSF tTau, pg/ml & $264(103.9)^{c}$ & $388.9(182.3)^{c}$ & $275.4(144.5)^{c}$ & $367.5(0.7)$ & $276.1(122.7)^{c}$ & $332.7(162)^{c}$ & $774.2(390.8)^{\mathrm{a}, \mathrm{b}, \mathrm{d}, \mathrm{f}, \mathrm{g}}$ \\
\hline CSF pTau, pg/ml & $39.8(17.8)^{c}$ & $45.7(16.1)^{c}$ & $42.8(21.9)^{c}$ & $47.2(10)$ & $33.9(15)^{c}$ & $42.1(16.4)^{c}$ & $121.2(71)^{a, b, d, f, g}$ \\
\hline
\end{tabular}

MMSE, Mini-Mental State Examination; pGFAP, plasma glial fibrillary acidic protein; pNfL, plasma neurofilament light chain; CN, cognitivelly normal participants; FTD, Frontotemporal dementia; AD, Alzheimer disease; bvFTD, behavioral variant of frontotemporal dementia; nfvPPA, nonfluent variant of primary progressive aphasia; sVPPA, semantic variante of primary progressive aphasia; PSP-CBD, progressive supranuclear palsy-corticobasal syndrome spectrum

${ }^{a}$ Different from Control $(P<0.05)$

${ }^{\mathrm{b}}$ Different from FTD $(P<0.05)$

c Different from AD $(P<0.05)$

${ }^{\mathrm{d}}$ Different from bvFTD $(P<0.05)$

${ }^{\text {e }}$ Different from fnvPPA $(P<0.05)$

${ }^{f}$ Different from svPPA $(P<0.05)$

${ }^{g}$ Different from PSP-CBD $(P<0.05)$. Data are shown as mean (standard deviation)

\section{Diagnostic accuracy of plasma biomarkers and their combination to differentiate between FTD, AD and CN}

We next explored the diagnostic performance of pGFAP, pNfL and their combination using logistic regression to discriminate across the different groups. The basic model included age, sex and APOEE4 allele status. The diagnostic performance of this basic model was then compared with a panel including the variables in the basic model together with pGFAP and pNfL. ROC curve analysis (Fig. 2) showed that the combination of pGFAP and pNfL had higher accuracy than the two plasma markers separately in differentiating FTD from AD (combination: AUC 0.78 [95\%CI 0.70-0.86]; pGFAP: AUC 0.7 [95\%CI 0.61-0.71]; pNfL: AUC 0.61 [95\%CI 0.51-0.71], $P=0.04$ and $P=0.002$, respectively) and for the discrimination between FTD and CN (combination: AUC 0.82 [95\%CI 0.75-0.89]; pGFAP: AUC 0.76 [95\%CI 0.68-0.84]; pNfL: AUC 0.81 [95\%CI 0.74-0.88], $P=0.02$ and $P=0.51$, respectively). None of the two plasma markers, individually or in combination, showed higher accuracy than the basic model. However, the addition of pGFAP and pNfL to the basic model (panel model) led to better diagnostic performance than the basic model both in differentiating FTD from AD (AUC 0.83 [95\%CI 0.75-0.9] vs AUC 0.69 [95\%CI 0.6-0.79], $P=0.003$ ) and $\mathrm{AD}$ from $\mathrm{CN}$ (AUC 0.96 [95\%CI 0.93-1] vs AUC 0.89 [95\%CI 0.83-0.95], $P=0.0007)$. For differentiation of FTD from $\mathrm{CN}$, the panel showed highest AUC compared to other methods (all $P<0.05$ ), but not compared to the basic model (AUC 0.88 [95\%CI 0.83-0.94] vs AUC 0.87 [95\%CI $0.81-0.93], P=0.08$ ).

Taking into account that pGFAP increases with age, we repeated the same analyses in an age-matched subgroup. In this new comparison, similar results were observed except in the comparison between FTD and $\mathrm{CN}$, in which both pNfL and the combination showed higher diagnostic performance compared to this basic model 

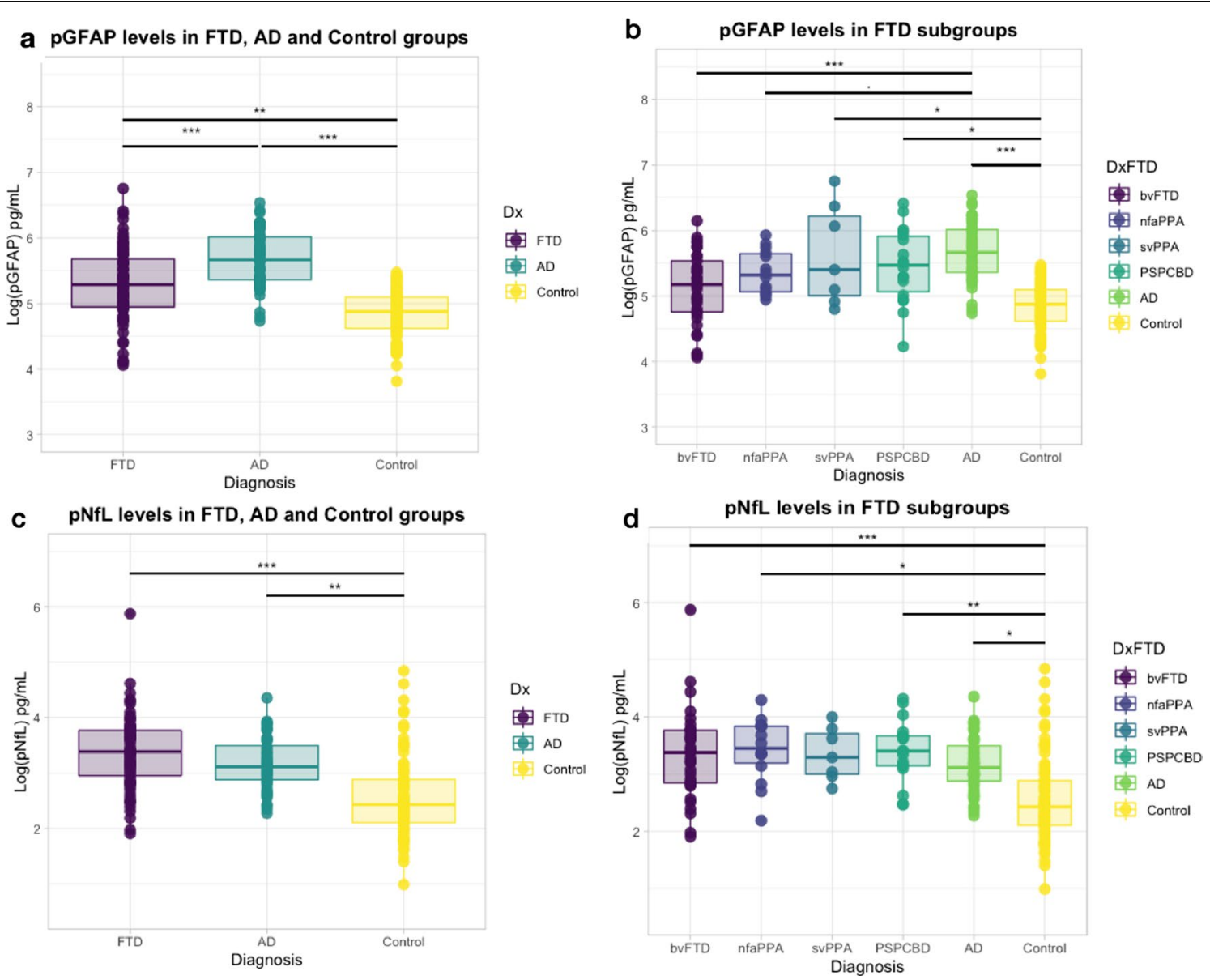

Fig. 1 Comparison of pGFAP and pNfL in different groups. a pGFAP in the FTD, AD and cognitively normal groups; $\mathbf{b}$ pGFAP concentration in all FTD subgroups, $A D$ and cognitively normal groups; $\mathbf{c}$ pNfL concentration in the FTD, AD and cognitively normal groups; $\mathbf{d}$ pNfL concentration in all FTD subgroups, AD and cognitively normal groups. ${ }^{*} P<0.05,{ }^{* *} P<0.01,{ }^{* * *} P<0.001$. pGFAP, plasma glial fibrillary acidic protein; pNfL, plasma neurofilament light chain; CN, cognitively normal participants; FTD, frontotemporal dementia; AD, Alzheimer's disease

(Combination: AUC 0.8 [95\%CI 0.7-0.9]; pNfL: AUC 0.8 [95\%CI 0.7-0.9]; Basic model: AUC 0.59 [95\%CI 0.470.72 ], $P=0.05$ and $P=0.005$ ) (Additional file 1 ).

\section{pGFAP correlated with other fluid biomarkers, cognitive and functional scores and neuroimaging}

In the whole sample, pGFAP level correlated with pNfL $($ Rho $=0.53, P<0.001)$, CSF NfL $($ Rho $=0.52, P<0.001)$ and CSF YKL-40 levels (Rho $=0.49, P<0.001$ ) after adjusting for age and sex. To avoid group effects on the correlation assessment, we studied correlations of these biomarkers within groups. The pGFAP level correlated with plasma and CSF NfL in FTD (Rho $=0.49, P<0.001$; Rho $=0.32, P<0.02)$ and in $\mathrm{CN}$ (Rho $=0.4, P<0.001$; Rho $=0.3, P=0.016$ ), but not with CSF YKL-40. By contrast, in the AD group, pGFAP level correlated with pNfL level only (Rho $=0.35, P=0.007)($ Fig. 3a-c).
The pGFAP level was significantly correlated with ADspecific CSF biomarkers, such as the A $\beta 42 / A \beta 40$ ratio, amyloid-PET centiloid, total tau and phosphorylated tau in the whole sample, but these correlations were not significant within diagnostic subgroups (Additional file 2).

After adjusting for age and sex, pGFAP was also significantly associated with the baseline MMSE scores in the whole sample (Rho $=-0.58, P<0.001)$ and in the FTD group (Rho $=-0.33, P=0.005)$, but not in the $\mathrm{AD}$ or the $\mathrm{CN}$ group (Fig. $3 \mathrm{~d}$ ). In the FTD group, after adjusting for age, higher pGFAP concentrations were associated with lower FTD-FRS scores $(r=-0.28, P=0.046$, Fig. 3e).

We assessed the correlation between pGFAP and cortical thickness in a subset of 122 participants (29 FTD, 25 $\mathrm{AD}$ and $68 \mathrm{CN}$ ) with structural MRI suitable for quantitative analyses. After adjusting for age, sex and neuroimaging acquisition center, vertex-wise regression analysis showed a significant association between pGFAP and 

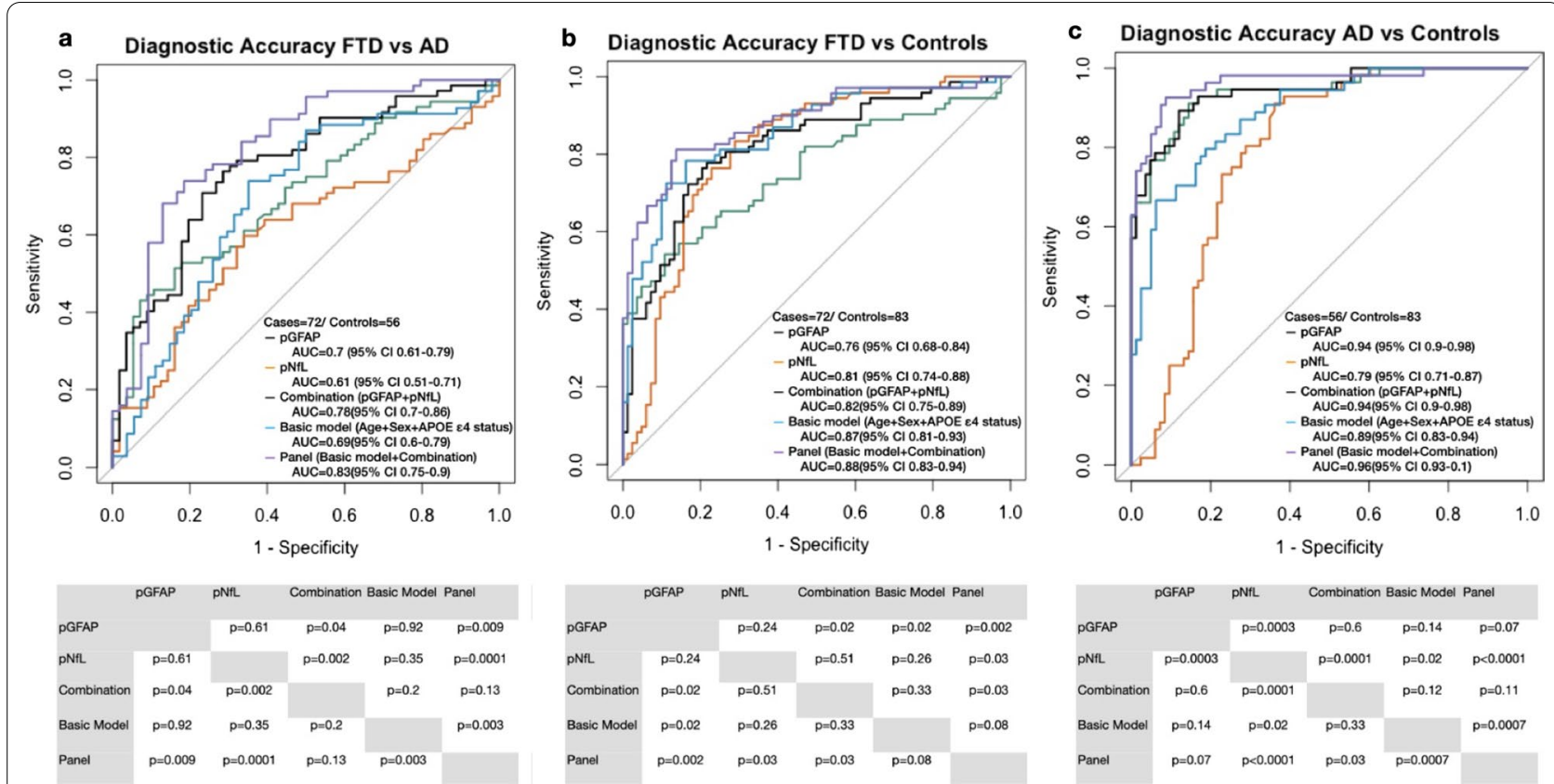

Fig. 2 Receiver operating characteristic curves (ROC) of pGFAP, plasma neurofilament light chain, their combination, a basic model with risk factors (age, sex and APOE), and a combination of plasma biomarkers with additional risk factors to discriminate FTD from AD (a), FTD from normal cognitively normal (b), and AD from cognitively normal (c). AUC, area under the curve; pGFAP, plasma glial fibrillary acidic protein; pNfL, plasma neurofilament light chain; CN, cognitively normal participants; FTD, frontotemporal dementia; AD, Alzheimer's disease

cortical thickness in the orbitofrontal and occipital pole regions (Fig. 3f, $\mathrm{P}<0.05$ ) in the FTD group. In the $\mathrm{AD}$ and the $\mathrm{CN}$ groups, no correlation between pGFAP concentration and cortical thickness was found (not shown).

\section{Baseline pGFAP level predicts cognitive decline}

To investigate the prognostic performance of pGFAP levels, we divided pGFAP levels in tertiles within each symptomatic group: low $(<154 \mathrm{pg} / \mathrm{ml}$ in FTD and $<228 \mathrm{pg} / \mathrm{ml}$ in AD), medium $(154-240 \mathrm{pg} / \mathrm{ml}$ in FTD and $228-366 \mathrm{pg} / \mathrm{ml}$ in AD) and high $(>240 \mathrm{pg} / \mathrm{ml}$ in FTD and $>366 \mathrm{pg} / \mathrm{ml}$ in AD).

Linear-mixed model analysis was used to assess the relationship between the baseline pGFAP level and cognitive decline measured by changes in MMSE score during follow-up. After adjusting for age, sex and baseline MMSE score, patients in the highest pGFAP tertile showed a greater change in MMSE score compared to those in the lowest tertile in both FTD (mean loss of 1.40 points annually, $P=0.003$ ) and $\mathrm{AD}$ (mean loss of 1.20 points annually, $P<0.001$ ) (Fig. 4a, b).

Kaplan Meier curves (Fig. 5) and Cox regression analyses including baseline age and sex as covariates were used to assess progression to moderate cognitive impairment (MMSE score $<20$ ). Compared to the lowest tertile, the highest tertile of pGFAP was associated with increased risk of poor cognitive outcome both in FTD and in AD
(1.40 points annually, $\mathrm{HR}=3.82$ in FTD; 1.20 points annually, $\mathrm{HR}=2.26$ in $\mathrm{AD}$, both $P<0.001$ ).

\section{Discussion}

In this study, we found that pGFAP was increased in FTD, and that its level differs from that in AD patients. pGFAP also correlated with neuronal damage biomarkers, cognitive and functional scores and structural imaging measures in FTD. Importantly, a higher pGFAP level was associated with a greater change in MMSE score during follow-up and predicted progression to moderate cognitive impairment in FTD. Our findings suggest that pGFAP could be a useful marker of disease severity and for prognostic assessment in FTD, in addition to its role in $\mathrm{AD}$.

Emerging evidence suggests that astrocytes play an essential role in neuroinflammation and are involved in the pathophysiology of several neurological diseases [34, 36, 38]. Biomarkers that track astrocyte biology, such as GFAP, have been investigated over the past few years in neurodegenerative diseases. In AD, previous studies have shown that reactive astrocytes are closely associated with senile plaques and neurofibrillary tangles [37-39]. Elevated concentrations of GFAP in CSF and blood have been observed in $\mathrm{AD}$, and it has been reported that its levels correlate with disease severity $[8-16,19]$. Recent studies have shown that the elevated CSF and plasma 


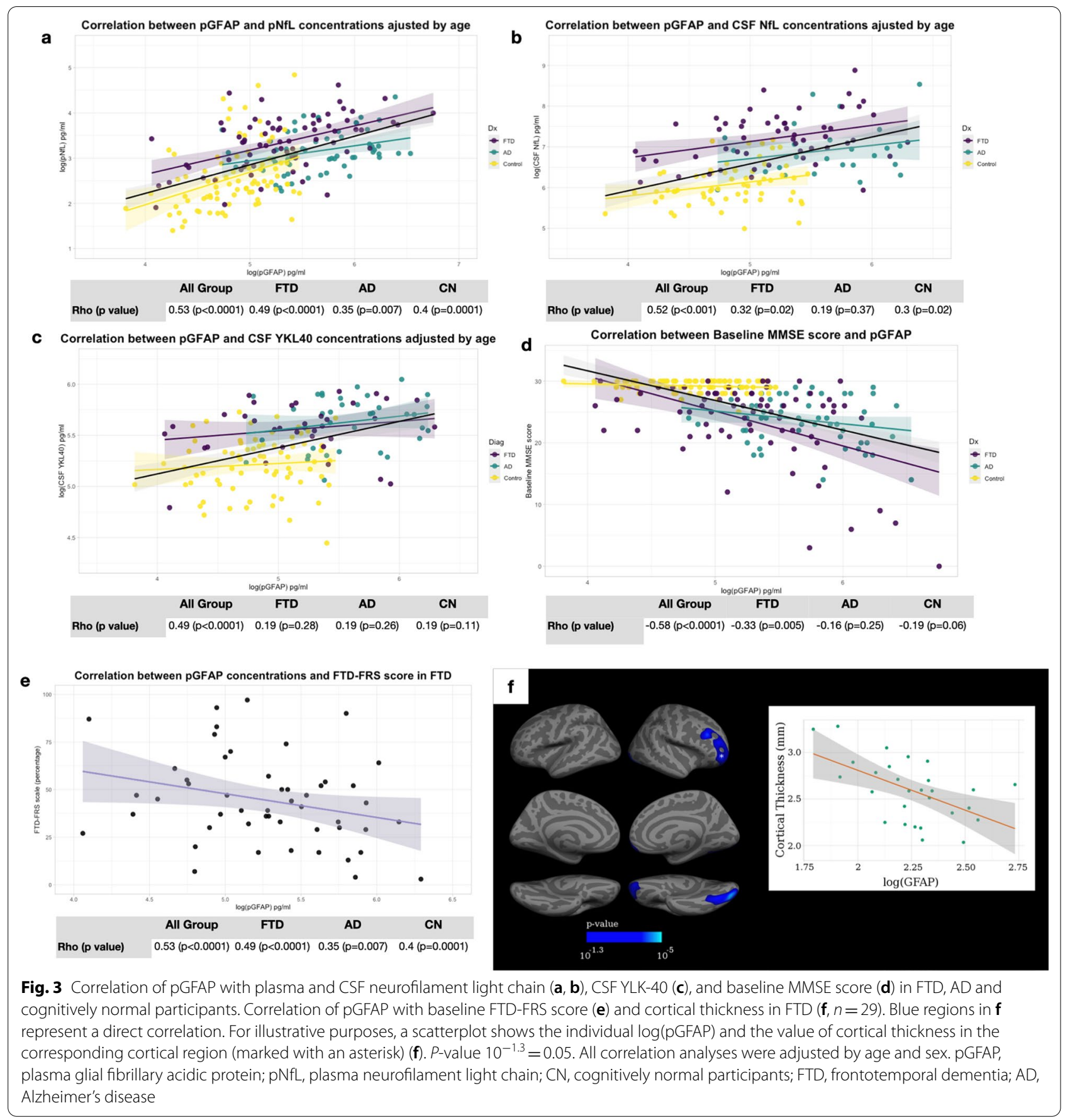

GFAP are associated with amyloid pathology, also in cognitively unimpaired subjects [15-20]. In addition, astrogliosis is also recognized in FTD. Astrogliosis is mainly confined to concrete regions such as the frontal cortex and the hippocampus [40, 41]. Interestingly, increased CSF and plasma GFAP levels have been reported in both sporadic and genetic FTD cohorts [10-12, 14, 23]. These findings suggest that pGFAP may be a valuable tool in
FTD diagnosis. According to this hypothesis, various studies have observed elevated pGFAP concentration in different FTD subgroups and both in presenile and lateonset bvFTD cases [24, 25, 27]. In addition, one study in genetic FTDs found increased pGFAP concentration in progranulin-associated symptomatic FTD patients [23]. Here we report an increased pGFAP level in FTD patients, which was, however, lower than that of AD. Our 

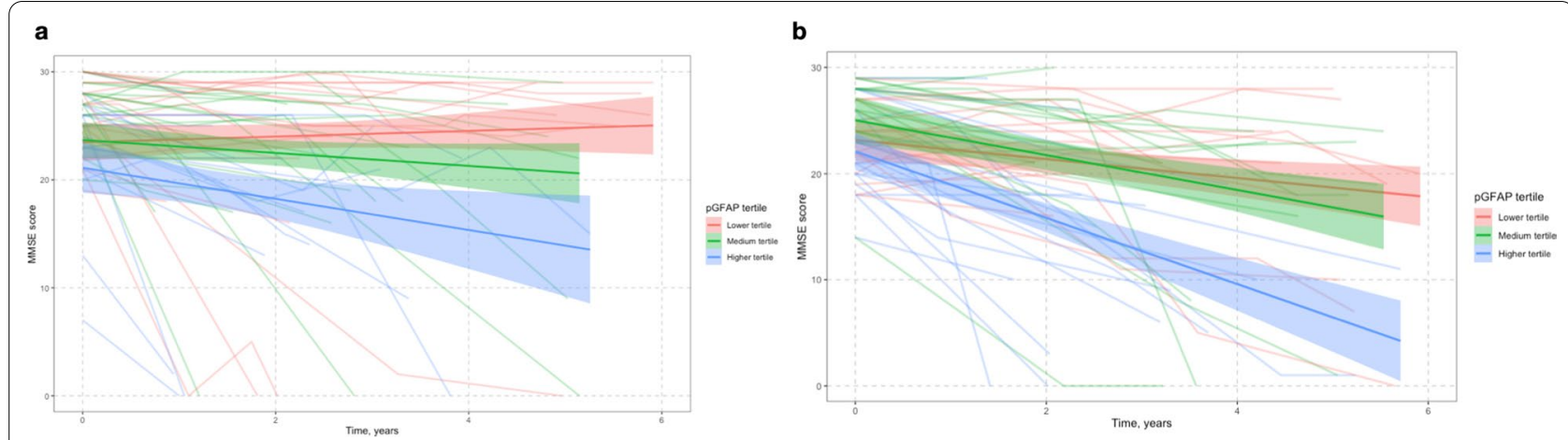

Fig. 4 Relationship between the baseline tertile of PGFAP and longitudinal changes of cognitive score (MMSE score) in FTD (a) and AD (b). Cognitive changes were estimated through linear mixed effects regression models adjusted for age and baseline MMSE score. Red lines show the lowest tertile (below $154 \mathrm{pg} / \mathrm{ml}$ in FTD and below $228 \mathrm{pg} / \mathrm{ml}$ in AD), green lines show the medium tertile (154-240 pg/ml in FTD and 226-366 pg/ $\mathrm{ml}$ in AD), and blue lines show the highest tertile of pGFAP level (above $240 \mathrm{pg} / \mathrm{ml}$ in FTD and above $366 \mathrm{pg} / \mathrm{ml}$ in AD). Shaded areas indicate the 95\% confidence interval for predicted cognitive scores. pGFAP, plasma glial fibrillary acidic protein; pNfL, plasma neurofilament light; CN, cognitivelly normal participants; FTD, frontotemporal dementia; AD, Alzheimer's disease
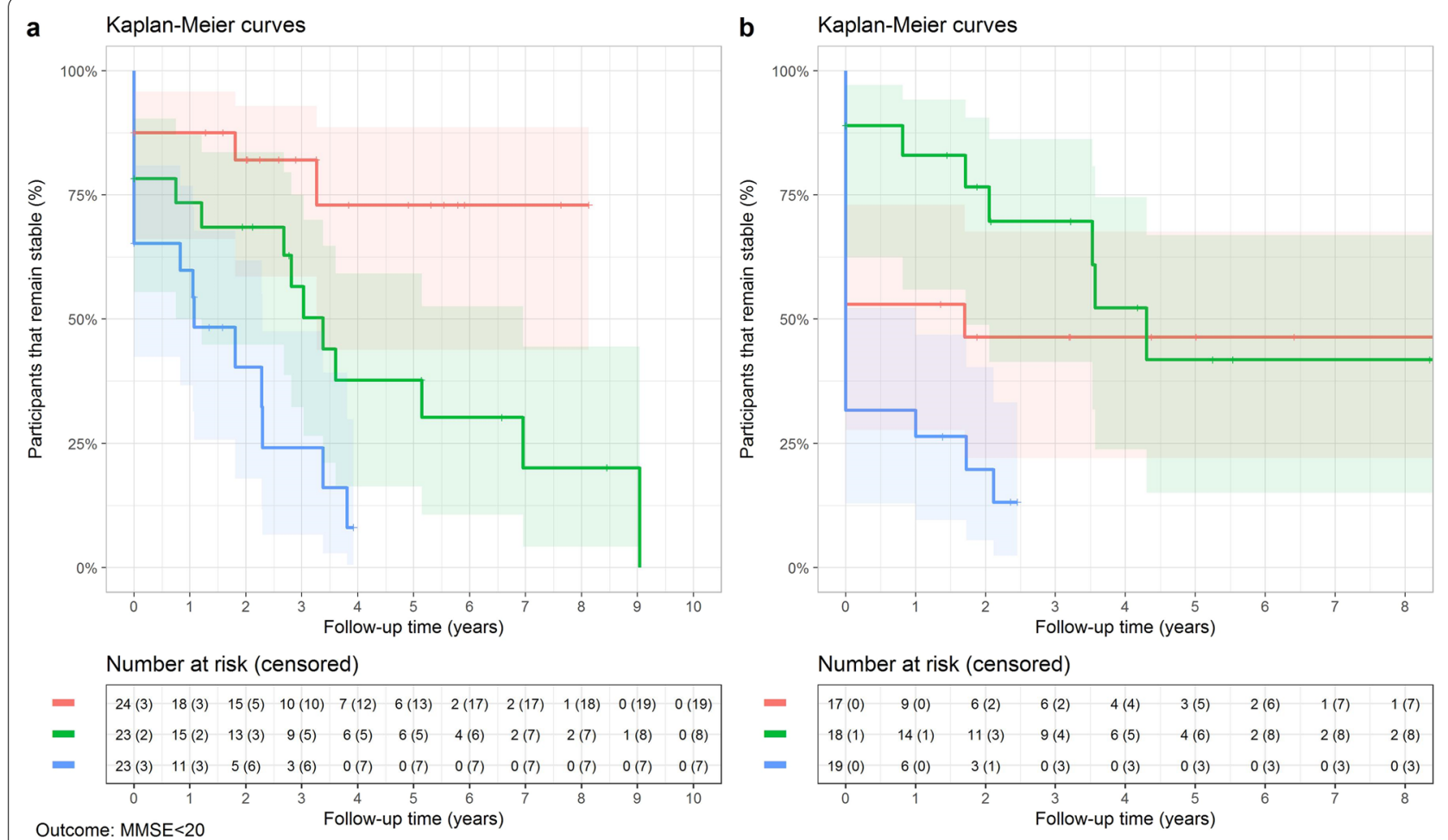

Number at risk (censored)

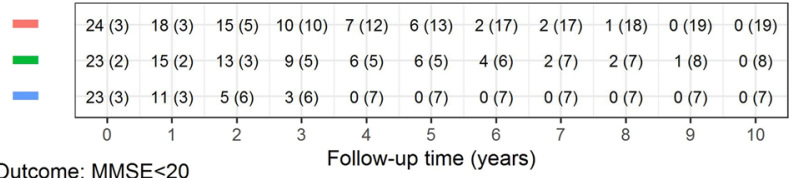

Outcome: MMSE<20

Fig. 5 Kaplan Meier curves of clinical progression to significant cognitive impairment (MMSE score <20) in FTD (a) and AD (b) individuals with low, medium or high baseline pGFAP level. Red lines show the lowest tertile (below $154 \mathrm{pg} / \mathrm{ml}$ in FTD and below $228 \mathrm{pg} / \mathrm{ml}$ in AD), green lines show medium tertile (154-240 pg/ml in FTD and 226-366 pg/ml in AD), and blue lines show the highest tertile of biomarker concentrations (above $240 \mathrm{pg} / \mathrm{ml}$ in FTD and above $366 \mathrm{pg} / \mathrm{ml}$ in AD). In FTD, the median time of progression to significant cognitive impairment was 3.4 (0.8-7.0) years in the medium-tertile pGFAP group and $1.1(0-2.3)$ years in the high-tertile pGFAP group (Log-rank test, $P<0.0001)$. pGFAP, plasma glial fibrillary acidic protein; FTD, frontotemporal dementia; AD, Alzheimer's disease

results are in line with previous reports of higher concentrations of pGFAP in AD compared to non-AD dementias $[13,18]$. However, other studies found no differences between groups [20, 24]. These discrepancies could be explained by the heterogeneity of the patient composition: some studies included different types of dementias 
in the non-AD dementia group and little was known about the function of pGFAP in such contexts, such as vascular dementia or dementia with Lewy bodies. Our results support previous clinical-pathological findings that in FTD, astrocytic activation is at a lower extent and is more confined to specific brain regions than that in $\mathrm{AD}[40,41]$. On the other hand, the increase in pNfL, a marker of axonal damage, suggests a similar degree of neurodegeneration in FTD compared to AD. One possible explanation for the differences in pGFAP levels but not in pNfL between FTD and AD is that FTD and AD are characterized by different neuroinflammatory patterns, with different degrees of astroglial activation in different subpopulations but leading to a similar degree of neuronal degeneration. Similar to a previous study [24], we found that the pGFAP level tended to be different across the subtypes of the FTD spectrum, being significantly higher in svFTD and PSP-CBD compared to CN. This finding could indicate differences in the degree of astrocytic degeneration in different clinical phenotypes [23].

The current study showed that pGFAP had an acceptable performance to discriminate FTD from AD $(\mathrm{AUC}=0.7)$ and $\mathrm{CN}(\mathrm{AUC}=0.76)$. These accuracies were somewhat lower than the values reported in two recent studies using pGFAP to identify bvFTD from $A D$ $(\mathrm{AUC}=0.85)$ and frontotemporal lobar degeneration $(F T L D)$ from primary psychiatric disorders $(A U C=0.82)$ $[13,27]$. These discrepancies could be attributed to differences in cohort characteristics and heterogeneity due to different composition of FTD subtypes. An interesting finding was that, contrary to pNfL, which tend to be higher in FTD compared to AD, the pGFAP level was significantly lower in FTD, suggesting the potential diagnostic utility of their combination. Indeed, the combination of pGFAP with pNfL improved the diagnostic performance in distinguishing FTD from $\mathrm{AD}$ and $\mathrm{CN}$. Moreover, the incorporation of age, sex and APOE\&4 allele status improved the diagnostic performance to an AUC of 0.83 in differentiating FTD from AD and an AUC of 0.89 in differentiating FTD from CN. In addition, pGFAP was particularly promising in identifying $A D$, consistent with previous observations [13, 15, 17-20]. Taken together, this study suggests that the combination of plasma astrocytic and neuronal markers could be relevant in FTD.

We observed that the pGFAP level increased with age in controls, similar to what has been observed in previous studies [13, 17, 23, 25], and we took age into account when interpreting this marker. Consistent with various recent reports [15, 17, 21, 23], pGFAP significantly correlated with CSF and plasma NfL, suggesting that glial activation and neuroaxonal degeneration are correlated.
Previous studies have reported a weak correlation between CSF GFAP and YKL-40 levels in neurodegenerative diseases $[11,12]$. Similarly, we did not find significant correlation between pGFAP and CSF YKL-40 levels. This finding supports the hypothesis that different astrocyte subpopulations or different spatial distributions are involved in the pathophysiology of FTD and AD [12, 13, 37, 38-41].

The association between elevated pGFAP level, disease severity and prognostic markers in $\mathrm{AD}$ has been reported before [15, 17-22]. Previous studies reported that the serum GFAP has potentials to predict future conversion to dementia in cognitively normal individuals, not only to $\mathrm{AD}$, but also to other dementias including FTD $[16,17$, 22]. However, pGFAP as a marker of disease severity and prognosis in FTD has seldom been investigated [13, 25, 26]. In the current study, after adjusting for age and sex, pGFAP remained significantly correlated with cognitive and imaging measures in FTD. Higher levels of pGFAP have been shown to be associated with lower brain volumes in GRN and C9orf72 presymptomatic carriers [23], and their association with smaller hippocampal volume in svPPA [26] and temporal atrophy in FTLD has been reported [27]. To our knowledge this is the first study to show that higher concentrations of pGFAP are also associated with orbitofrontal cortical thickness in the whole FTD group. In line with other reports in AD patients [16, $20,22]$, we observed that pGFAP predicted faster cognitive decline both in FTD and AD. This is the first report of the association between pGFAP and cognitive decline in FTD; we found a higher rate of cognitive decline among those with pGFAP level $>240 \mathrm{pg} / \mathrm{ml}$. Surprisingly, the relationship between pGFAP and disease severity in $\mathrm{AD}$ was not confirmed in our cohort. One of the considerations when comparing our study with the previous ones is that other studies included both $A \beta+$ and $A \beta$ - subjects $[15,22]$, whereas in our study the correlation analysis was performed in disease subgroups. Previous studies have found that pGFAP is increased in early stages of $A D$ [15-22] and does not differ between disease stages [19, 20], which could explain the lack of association with imaging measures in the AD subgroup. Consistent with previous studies [2, 7, 17], our results show that the glial biomarker increases also in FTD, in addition to $\mathrm{AD}$, and its level increases later in the disease course in FTD than in AD, when the disease is more advanced.

The strength of our study is the relatively large sample size, the inclusion of several FTD subgroups, regular follow-up of the participants and the use of multimodal approach, with inclusion of clinical measures, plasma and CSF assessments, and structural imaging. Additionally, all blood-based biomarkers were collected using the same standard operating procedures and measured following 
a harmonized protocol. This study also has some limitations, in that it did not include serial longitudinal measures of pGFAP, lacked neuropathological confirmation, and excluded AD copathologies in FTD groups.

\section{Conclusion}

In conclusion, our study shows that the pGFAP concentration may be useful in FTD and could improve its diagnosis. Furthermore, our data support that pGFAP is not an exclusive marker in AD, but also plays an essential role in other amyloid-independent processes involved in FTD. pGFAP could be used as a marker for FTD severity and prognosis.

\begin{abstract}
Abbreviations
AD: Alzheimer's disease; AUC: Area under the curve; bvFTD: Behavioral variant of frontotemporal dementia; CN: Cognitively normal participants; CSF: Cerebrospinal fluid; FTD: Frontotemporal dementia; FTLD: Frontotemporal lobar degeneration; FTD-FRS: Frontotemporal Dementia Rating Scale; GFAP: Glial fibrillary acidic protein; HR: Hazard ratio; MMSE: Mini-Mental State Examination; NfL: Neurofilament light chain; nfvPPA: Nonfluent variant of primary progressive aphasia; PET: Positron emission tomography; PGFAP: Plasma Glial fibrillary acidic protein; pNfL: Plasma neurofilament-light; PSP-CBD: Progressive supranuclear palsy-corticobasal syndrome spectrum; ROC: Receiver operating characteristic; SPIN: Sant Pau Initiative on Neurodegeneration; sVPPA: Semantic variant of primary progressive aphasia.
\end{abstract}

\section{Supplementary Information}

The online version contains supplementary material available at https://doi. org/10.1186/s40035-021-00275-w.

Additional file 1. Diagnostic accuracy of plasma biomarkers and their combination to differentiate between FTD, AD and CN in age-matched group

Additional file 2. The correlation of pGFAP with CSF AD-specific biomarkers

\section{Acknowledgements}

The authors would like to acknowledge the Department of Medicine at the Universitat Autònoma de Barcelona. We are grateful to all participants in the study and their families. We also thank all the clinical team members who were involved in the selection and assessment of participants in the SPIN cohort, and the laboratory and neuroimaging teams for sample handling, biomarker analyses, and structural support.

\section{Authors' contributions}

$N Z, D A$ and $A L$ designed the study. NZ, MS, IIG, VM, TE, IB, MA, JA, LM, OB, IS, MBSS, AS, LV, JP, RB, JC, MCI, JF, AL and DA acquired data relevant for the study. $N Z, D A$ and VM performed statical analysis. NZ, DA and AL contributed in analysis and interpretation of data. DA and AL participated in study supervision or coordination. NZ, DA, AL drafted the first version of the manuscript. All authors read and approved the final manuscript.

\section{Funding}

This study was supported by the Fondo de Investigaciones Sanitario (FIS), Instituto de Salud Carlos III (PI14/01126, PI17/01019 and PI20/01473 to JF, PI13/01532 and PI16/01825 to RB, PI18/00335 to MCI, PI18/00435 and INT19/00016 to DA, PI17/01896 and AC19/00103to AL) and the CIBERNED program (Program 1, Alzheimer Disease to AL), jointly funded by Fondo Europeo de Desarrollo Regional, Unión Europea, "Una manera de hacer Europa". This work was also supported by Generalitat de Catalunya (2017-SGR-547, SLT006/17/125 to DA, SLT006/17/119 to JF, SLT002/16/408 to AL) and "Marató
TV3" foundation grants 20141210 to JF, 044412 to RB and 20142610 to AL. This work was also supported by a grant from the Fundació Bancaria La Caixa to RB (DABNI project). The sponsors of the study did not take part in the design and conduct of the study; collection, management, analysis and interpretation of the data; writing and review of the report; or the decision to submit the article for publication.

\section{Availability of data and materials}

The datasets used and/or analyses during the current study are available from the corresponding author on reasonable request.

\section{Declarations}

Ethics approval and consent to participate

All procedures in the study were approved by the Hospital de Sant Pau ethics committee. All participants provided written informed consent.

\section{Consent for publication}

Not applicable.

\section{Competing interest}

Nuole Zhu is employed by Hospital de la Santa Creu i Sant Pau. Declarations of interest: none. Miguel Santos is employed by Hospital de la Santa Creu i Sant Pau. He is funded by a "Juan Rodés" research grant from the Institute of Health Carlos III. Ignasio Illán-Gala is supported by the Global Brain Health Institute (Atlantic Fellow for Equity in Brain Health and pilot award for global brain health leaders CBHI ALZ UK-21-720973) and the "Juan Rodés" grant from the Institute of Health Carlos III /JR20/00018). Victor Montal is employed by the Biomedical Research Institute Sant Pau. Declarations of interest: none. Teresa Estellés is employed by Biomedical Research Institute Sant Pau. Declarations of interest: Dr. Estellés is funded by a "Río Hortega" research grant from the Institute of Health Carlos III. Isabel Barroeta is employed by Hospital de la Santa Creu i Sant Pau. Declarations of interest: none. Miren Altuna is employed by Biomedical Research Institute Sant Pau. Declarations of interest: Dr. Estellés is funded by a "Río Hortega" research grant from the Institute of Health Carlos III. Javier Arranz is employed by Biomedical Research Institute Sant Pau. Declarations of interest: none. Laia Muñoz is employed by Biomedical Research Institute Sant Pau. Declarations of interest: none. Olivia Belbin is employed by Biomedical Research Institute Sant Pau. Dr. Belbin is funded by a "Miguel Servet" research grant from the Institute of Health Carlos III. Isabel Sala is employed by Hospital de la Santa Creu i Sant Pau. Declarations of interest: none. M. Belén Sánchez-Saudinós is employed by the Biomedical Research Institute Sant Pau. Declarations of interest: none. Andrea Subirana is employed by the Biomedical Research Institute Sant Pau. Declarations of interest: none. Laura Videla is employed by Fundació Catalana Síndrome de Down. Declarations of interest: none. Jordi Pegueroles is employed by the Biomedical Research Institute Sant Pau. Declarations of interest: none. Rafael Blesa is employed by Hospital de la Santa Creu i Sant Pau and received research grants from Institute of Health Carlos III, Fundació Bancària Obra Social La Caixa and Fundació La Marató de TV3. He participated in advisory boards from Lilly and Nutricia, and he received speakers honoraria and travel funding from Novartis and Nutricia. Jordi Clarimón is employed by Biomedical Research Institute Sant Pau and received research grants from Generalitat de Catalunya and from Institute of Health Carlos III. Maria Carmona-Iragui is employed by Hospital de la Santa Creu i Sant Pau. Declarations of interest: none. Juan Fortea is employed by Hospital de la Santa Creu i Sant Pau and received research grants from Institute of Health Carlos III, Fundació La Marató de TV3, and Pla Estratègic de Recerca i Innovació en Salut (PERIS). Alberto LLeó is employed by Hospital de la Santa Creu i Sant Pau and received research grants from CIBERNED, Institute of Health Carlos III and Fundació BBVA. He participated in advisory boards from Fujirebio-Europe, Nutricia, Biogen and received speaker honoraria from Lilly. Daniel Alcolea is employed by Hospital de la Santa Creu i Sant Pau and received research grants from Pla Estratègic de Recerca i Innovació en Salut (PERIS SLT006/17/125), and from Institute of Health Carlos III (PI18/00435 and INT19/00016). He participated in advisory boards from Fujirebio-Europe and Roche Diagnostics and received speaker honoraria from Fujirebio-Europe, Roche Diagnostic, Nutricia, Esteve and from Krka Farmacéutica S.L. 


\begin{abstract}
Author details
'Sant Pau Memory Unit, Department of Neurology, Institut d'Investigacions Biomèdiques Sant Pau - Hospital de Sant Pau, Universitat Autònoma de Barcelona, 08041 Barcelona, Spain. ${ }^{2}$ Centro de Investigación Biomédica en Red en Enfermedades Neurodegenerativas (CIBERNED), 28031 Madrid, Spain. ${ }^{3}$ Autonomous University of Barcelona, 08913 Barcelona, Spain. ${ }^{4}$ Fundación Catalana Síndrome de Down, Centre Mèdic Down, 08029 Barcelona, Spain.
\end{abstract}

Received: 7 October 2021 Accepted: 1 December 2021 Published online: 10 December 2021

\section{References}

1. Rohrer JD, Woollacott IO, Dick KM, Brotherhood E, Gordon E, Fellows A, et al. Serum neurofilament light chain protein is a measure of disease intensity in frontotemporal dementia. Neurology. 2016;87(13):1329-36.

2. Alcolea D, Vilaplana E, Suárez-Calvet M, Illán-Gala I, Blesa R, Clarimón J, et al. CSF SAPP $3, Y K L-40$, and neurofilament light in frontotemporal lobar degeneration. Neurology. 2017;89(2):178-88.

3. Meeter LHH, Vijverberg EG, Del Campo M, Rozemuller AJM, Donker Kaat L, de Jong FJ, et al. Clinical value of neurofilament and phosphotau/tau ratio in the frontotemporal dementia spectrum. Neurology. 2018:90(14):e1231-9.

4. Bridel C, van Wieringen WN, Zetterberg H, Tijms BM, Teunissen CE; and the NFL Group, et al. Diagnostic value of cerebrospinal fluid neurofilament light protein in neurology: a systematic review and meta-analysis. JAMA Neurol. 2019;76(9):1035-48.

5. van der Ende EL, Meeter LH, Poos JM, Panman JL, Jiskoot LC, Dopper EGP, et al. Genetic Frontotemporal dementia Initiative (GENFI). Serum neurofilament light chain in genetic frontotemporal dementia: a longitudinal, multicentre cohort study. Lancet Neurol. 2019;18(12):1103-11.

6. Ashton NJ, Janelidze S, Al Khleifat A, Leuzy A, van der Ende EL, Karikari TK, et al. A multicentre validation study of the diagnostic value of plasma neurofilament light. Nat Commun. 2021;12(1):3400.

7. Alcolea D, Martínez-Lage P, Sánchez-Juan P, Olazarán J, Antúnez C, Izagirre $A$, et al. Amyloid precursor protein metabolism and inflammation markers in preclinical Alzheimer disease. Neurology. 2015;85(7):626-33.

8. Fukuyama R, Izumoto T, Fushiki S. The cerebrospinal fluid level of glial fibrillary acidic protein is increased in cerebrospinal fluid from Alzheimer's disease patients and correlates with severity of dementia. Eur Neurol. 2001:46(1):35-8.

9. Jesse S, Steinacker P, Cepek L, von Arnim CA, Tumani H, Lehnert S, et al. Glial fibrillary acidic protein and protein S-100B: different concentration pattern of glial proteins in cerebrospinal fluid of patients with Alzheimer's disease and Creutzfeldt-Jakob disease. J Alzheimers Dis. 2009;17(3):541-51.

10. Ishiki A, Kamada M, Kawamura Y, Terao C, Shimoda F, Tomita N, et al. Glial fibrillar acidic protein in the cerebrospinal fluid of Alzheimer's disease, dementia with Lewy bodies, and frontotemporal lobar degeneration. J Neurochem. 2016;136(2):258-61.

11. Abu-Rumeileh S, Steinacker P, Polischi B, Mammana A, Bartoletti-Stella

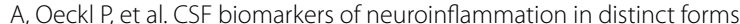
and subtypes of neurodegenerative dementia. Alzheimers Res Ther 2019;12(1):2.

12. Oeckl P, Weydt P, Steinacker $P$, Anderl-Straub S, Nordin F, Volk AE, et al. Different neuroinflammatory profile in amyotrophic lateral sclerosis and frontotemporal dementia is linked to the clinical phase. J Neurol Neurosurg Psychiatry. 2019:90(1):4-10.

13. Oeckl P, Halbgebauer S, Anderl-Straub S, Steinacker P, Huss AM, Neugebauer $\mathrm{H}$, et al. Glial fibrillary acidic protein in serum is increased in Alzheimer's disease and correlates with cognitive impairment. J Alzheimers Dis. 2019;67(2):481-8

14. Elahi FM, Casaletto KB, La Joie R, Walters SM, Harvey D, Wolf A, et al. Plasma biomarkers of astrocytic and neuronal dysfunction in early- and late-onset Alzheimer's disease. Alzheimers Dement. 2020;16(4):681-95.

15. Verberk IMW, Thijssen E, Koelewijn J, Mauroo K, Vanbrabant J, de Wilde A, et al. Combination of plasma amyloid beta ${ }_{(1-42 / 1-40)}$ and glial fibrillary acidic protein strongly associates with cerebral amyloid pathology. Alzheimers Res Ther. 2020:12(1):118.
16. Milà-Alomà M, Salvadó G, Gispert JD, Vilor-Tejedor N, Grau-Rivera O, Sala-Vila A, et al. Amyloid beta, tau, synaptic, neurodegeneration, and glial biomarkers in the preclinical stage of the Alzheimer's continuum. Alzheimers Dement. 2020;16(10):1358-71.

17. Chatterjee P, Pedrini S, Stoops E, Goozee K, Villemagne VL, Asih PR, et al. Plasma glial fibrillary acidic protein is elevated in cognitively normal older adults at risk of Alzheimer's disease. Transl Psychiatry. 2021;11(1):27.

18. Benedet AL, Milà-Alomà M, Vrillon A, Ashton NJ, Pascoal TA, Lussier F, et al. Differences between plasma and cerebrospinal fluid glial fibrillary acidic protein levels across the Alzheimer disease continuum. JAMA Neurol. 2021:e213671.

19. Cicognola C, Janelidze S, Hertze J, Zetterberg H, Blennow K, MattssonCarlgren N, et al. Plasma glial fibrillary acidic protein detects Alzheimer pathology and predicts future conversion to Alzheimer dementia in patients with mild cognitive impairment. Alzheimers Res Ther. 2021;13(1):68

20. Pereira JB, Janelidze S, Smith R, Mattsson-Carlgren N, Palmqvist S, Teunissen $C E$, et al. Plasma GFAP is an early marker of amyloid- $\beta$ but not tau pathology in Alzheimer's disease. Brain. 2021:awab223.

21. Verberk IM, Laarhuis MB, van den Bosch KA, Ebenau JL, van Leeuwenstijn M, Prins ND, et al. Serum markers glial fibrillary acidic protein and neurofilament light for prognosis and monitoring in cognitively normal older people: a prospective memory clinic-based cohort study. Lancet Healthy Long. 2021;179:1373-84.

22. Rajan KB, Aggarwal NT, McAninch EA, Weuve J, Barnes LL, Wilson RS, et al. Remote blood biomarkers of longitudinal cognitive outcomes in a population study. Ann Neurol. 2020;88(6):1065-76.

23. Heller C, Foiani MS, Moore K, Convery R, Bocchetta M, Neason M, et al. Plasma glial fibrillary acidic protein is raised in progranulinassociated frontotemporal dementia. J Neurol Neurosurg Psychiatry. 2020:91(3):263-70.

24. Marelli C, Hourregue C, Gutierrez LA, Paquet C, Menjot de Champfleur N, De Verbizier D, et al. Cerebrospinal fluid and plasma biomarkers do not differ in the presenile and late-onset behavioral variants of frontotemporal dementia. J Alzheimers Dis. 2020;74(3):903-11.

25. Benussi A, Ashton NJ, Karikari TK, Gazzina S, Premi E, Benussi L, et al. Serum glial fibrillary acidic protein (GFAP) is a marker of disease severity in frontotemporal lobar degeneration. J Alzheimers Dis. 2020;77(3):1129-41.

26. Heller C, Chan E, Foiani MS, Todd E, Russell LL, Greaves CV, et al. Plasma glial fibrillary acidic protein and neurofilament light chain are measures of disease severity in semantic variant primary progressive aphasia. J Neurol Neurosurg Psychiatry. 2020:jnnp-2020-325085.

27. Katisko K, Cajanus A, Huber N, Jääskeläinen O, Kokkola T, Kärkkäinen $V$, et al. GFAP as a biomarker in frontotemporal dementia and primary psychiatric disorders: diagnostic and prognostic performance. J Neurol Neurosurg Psychiatry. 2021;92(12):1305-12.

28. Alcolea D, Clarimón J, Carmona-Iragui M, Illán-Gala I, Morenas-Rodríguez E, Barroeta I, et al. The Sant Pau Initiative on Neurodegeneration (SPIN) cohort: a data set for biomarker discovery and validation in neurodegenerative disorders. Alzheimers Dement (N Y). 2019;5:597-609.

29. Rascovsky K, Hodges JR, Knopman D, Mendez MF, Kramer JH, Neuhaus J, et al. Sensitivity of revised diagnostic criteria for the behavioural variant of frontotemporal dementia. Brain. 2011;134(Pt 9):2456-77.

30. Gorno-Tempini ML, Hillis AE, Weintraub S, Kertesz A, Mendez M, Cappa SF, et al. Classification of primary progressive aphasia and its variants. Neurology. 2011;76(11):1006-14.

31. Armstrong MJ, Litvan I, Lang AE, BakTH, Bhatia KP, Borroni B, et al. Criteria for the diagnosis of corticobasal degeneration. Neurology. 2013;80(5):496-503.

32. Höglinger GU, Respondek G, Stamelou M, Kurz C, Josephs KA, Lang AE, et al. Clinical diagnosis of progressive supranuclear palsy: The movement disorder society criteria. Mov Disord. 2017;32(6):853-64.

33. Alcolea D, Pegueroles J, Muñoz L, Camacho V, López-Mora D, FernándezLeón A, et al. Agreement of amyloid PET and CSF biomarkers for Alzheimer's disease on Lumipulse. Ann Clin Transl Neurol. 2019;6(9):1815-24.

34. Kwon HS, Koh SH. Neuroinflammation in neurodegenerative disorders: the roles of microglia and astrocytes. Transl Neurodegener. 2020;9(1):42.

35. Fischl B, Dale AM. Measuring the thickness of the human cerebral cortex from magnetic resonance images. Proc Natl Acad Sci U S A. 2000;97(20):11050-5. 
36. Liddelow SA, Barres BA. Reactive astrocytes: production, function, and therapeutic potential. Immunity. 2017:46(6):957-67.

37. Beach TG, Walker R, McGeer EG. Patterns of gliosis in Alzheimer's disease and aging cerebrum. Glia. 1989;2(6):420-36.

38. Delacourte A. General and dramatic glial reaction in Alzheimer brains. Neurology. 1990;40(1):33-7.

39. Ross GW, O'Callaghan JP, Sharp DS, Petrovitch H, Miller DB, Abbott RD, et al. Quantification of regional glial fibrillary acidic protein levels in Alzheimer's disease. Acta Neurol Scand. 2003;107(5):318-23.

40. Englund E, Brun A. Frontal lobe degeneration of non-Alzheimer type. IV. White matter changes. Arch Gerontol Geriatr. 1987;6(3):235-43.

41. Arnold SE, Han LY, Clark CM, Grossman M, Trojanowski JQ. Quantitative neurohistological features of frontotemporal degeneration. Neurobiol Aging. 2000;21(6):913-9

- fast, convenient online submission

- thorough peer review by experienced researchers in your field

- rapid publication on acceptance

- support for research data, including large and complex data types

- gold Open Access which fosters wider collaboration and increased citations

- maximum visibility for your research: over $100 \mathrm{M}$ website views per year

At BMC, research is always in progress.

Learn more biomedcentral.com/submissions 